\title{
Estimation of Heat Transfer of a Front-heating Front-detection Laser Flash Method Measuring Thermal Conductivity for Silicate Melts at High Temperatures
}

\author{
Hiromichi OHTA, ${ }^{1)}$ Hiroyuki SHIBATA ${ }^{21}$ and Takeshi KASAMOTO ${ }^{11}$ \\ 1) Department of Materials Science, Ibaraki University, Hitachi 316-8511 Japan. E-mail: ohta@mx.ibaraki.ac.jp \\ 2) Institute of Multidisciplinary Research for Advanced Materials, Tohoku University, Sendai 980-8577 Japan.
}

(Received on September 27, 2005; accepted on November 17, 2005)

\begin{abstract}
The thermal conductivity of silicate melts at high temperatures was determined by applying a differential three-layered laser flash method. In this method, the correction for radiative heat transfer was indispensable to obtain accurate thermal conductivity of the melts at high temperatures because, at $1500 \mathrm{~K}$, the uncorrected values are 20 to $40 \%$ larger than the corrected ones; further, the corrected values strongly depend on the absorption coefficient of silicate melts. Recently, we applied the front-heating front-detection laser flash method using a short initial time period immediately after laser pulse irradiation for silicate melts. This method was considered to be unaffected by the radiative heat transfer. However, the amount of the radiative effect on thermal conductivity was not estimated. On the basis of heat transfer theory, the temperature responses of this method were numerically calculated by considering radiative heat transfer. The result revealed that the radiative effect is less than $4.4 \%$ even at $1743 \mathrm{~K}$.
\end{abstract}

KEY WORDS: thermal diffusivity; oxide melts; laser flash method; radiative heat transfer; conductive heat transfer; slag; silicate melts.

\section{Introduction}

The laser flash method is a useful method for measuring the thermal diffusivity of materials. It was originally developed for solid samples. ${ }^{1)}$ A pulse laser irradiates the front surface of a sample. The resultant temperature response of the other surface (rear surface) is measured. The measured temperature response curve is used to determine thermal diffusivity of the sample. This conventional front-heating rear-detection laser flash method was extended to measure the thermal diffusivity of melts using a three-layered cell at high temperatures. ${ }^{2-12)}$ For the application of the laser flash method at high temperatures, the correction for radiation is usually indispensables. The effect of radiation is an often complicated function of the physicalproperties of the sample and rather large even at relatively low temperatures. ${ }^{6,7)}$ The measurement of the optical properties of melts is also required to correct the radiative effect. ${ }^{6,7,13)}$

In order to overcome these difficulties, a front-heating front-detection laser flash method using an initial time period has been developed. ${ }^{14,15)}$ Figure 1 shows the schematic diagram of this method. In this setup, the effect of radiation might be small for the following reasons. The conductive heat flow is proportional to the temperature gradient, while the radiative heat flow is proportional to the temperature rise. Immediately after pulse irradiation, a steep temperature gradient is realized near the irradiated surface. The heat transfer in this region is detected by measuring the temperature response of the irradiated surface.
The theoretical temperature response of the irradiated surface, $\theta$, can be given by Eq. (1) under the following conditions. $^{16,17)}$

1. The system is in thermal equilibrium condition before laser irradiation.

2. The pulse duration of the laser is negligible.

3. The heat flow is one-dimensional.

4. The melt layer is semi-infinite.

5. The contact thermal resistance of the crucible/melt interface is negligible.

6. The temperature distribution in a platinum layer is isothermal because the platinum crucible is thin and its thermal diffusivity value is considerably larger than that of oxide layer presently investigated.

7. No radiative heat flow is considered.

$$
\theta=\theta_{\mathrm{M}} \exp \left(h^{2} t\right) \operatorname{erfc}(h \sqrt{t})
$$

where

$$
h=b_{\mathrm{s}} /\left(\rho_{\mathrm{d}} c_{\mathrm{pd}} l_{\mathrm{d}}\right)
$$

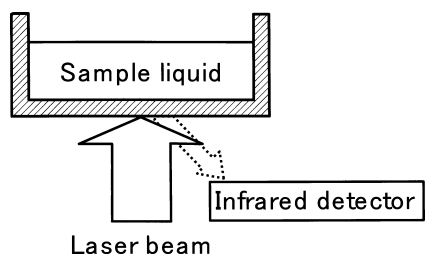

Fig. 1. Schematic diagram of cell of front-heating front-detection initial time laser flash method. 


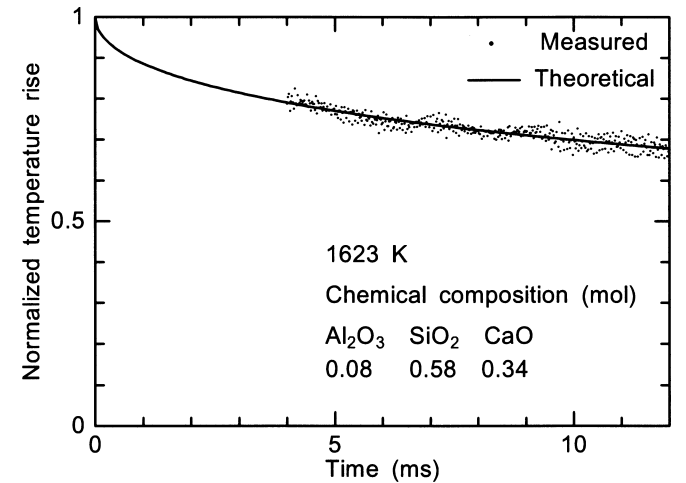

Fig. 2. Temperature response for molten silicate and that of Eq. (1) derived by fitting procedure. ${ }^{20)}$

$$
b_{\mathrm{s}}=\sqrt{\alpha_{\mathrm{s}}} \rho_{\mathrm{s}} c_{\mathrm{ps}}=\sqrt{\lambda_{\mathrm{s}} \rho_{\mathrm{s}} c_{\mathrm{ps}}}
$$

Here, $\theta_{\mathrm{M}} c_{\mathrm{p}}, \rho, b_{\mathrm{s}}$, and $l_{\mathrm{d}}$ represent the maximum temperature rise of the temperature response, specific heat, density, thermal effusivity, and thickness of platinum crucible, respectively. The subscripts of $d$ and $s$ indicate platinum crucible and slag, respectively. Here, time $t$ is defined as the elapsed time after irradiating a laser pulse. The values of $\theta_{\mathrm{M}}$ and $h$ can be estimated by fitting Eq. (1) to the measured temperature decay using the least square method, as shown in Fig. 2. The thermal diffusivity value of the slag is obtained from Eq. (3). For the laser flash method, it is well known that the measured temperature responses do not agree with the theoretical ones immediately after the laser irradiation because of the laser duration. ${ }^{18)}$ The thermal diffusivity of the platinum layer also affects the temperature response in this time region. Based on the results derived by the simulation of the temperature response curve in the front-heating front-detection laser flash method, the two optimum conditions for the data processing were established to avoid these problems. ${ }^{15}$ (1) The origin of time was $1.07 \mathrm{~ms}$ after the laser started. (2) The period of the analysis was between $4 \mathrm{~ms}$ and $12 \mathrm{~ms}$. Under these conditions, all the data analysis are carried out in this work.

Another major advantage of this method over the threelayered method is that the slag layer is openended. The cell setting is easy. The direct observation of liquid and the layout suitable for the degassing are essential to avoid the effect of bubbles that is one of the most difficult problems for the measurement of silicate at high temperatures. This method has been successfully employed for silicate melts at high temperatures. ${ }^{14,15,19,20)}$ The correction of radiative heat transfer was considered dispensable and a small radiative effect on measurement was suggested. ${ }^{20)}$ However, a quantitative discussion was not available.

The purpose of this work is to evaluate the effect of radiation through an accurate quantitative analysis of the temperature response derived by a numerical calculation of the heat transfer equations.

\section{Radiative and Conductive Heat Transfer}

The radiative heat transfer is a complicated nonlinear function of the temperature distribution. The problems of combined radiative conductive heat transfer are usually

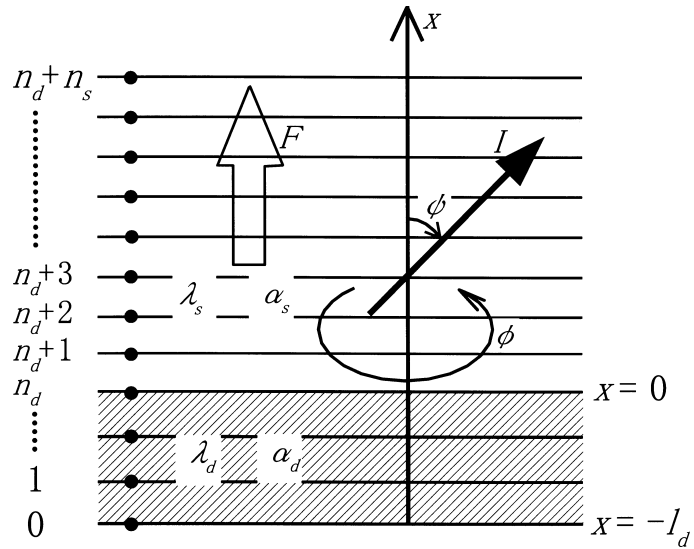

Fig. 3. Schematic diagram radiative heat flow in the front-heating front-detection laser flash method for melts.

solved numerically. In this section, we consider the basic equations according to Darby ${ }^{4)}$ and Siegel and Howell ${ }^{21)}$ for radiation. For conduction, simple boundary and initial conditions are employed as described in the following sections to make the effect of radiation clear.

Figure 3 shows a model for the heat transfer in the frontheating front-detection laser flash method. The layers are considered as infinite planes. The heat propagates in layers essentially in one dimension along the positive $x$ axis.

\subsection{In the Slag Layer}

The heat transfer equation for the temperature distribution $T(x, t)$ in the slag layer with the thermal conductivity $\lambda$ is

$$
\rho c_{\mathrm{p}} \frac{\partial T(t, x)}{\partial t}=\lambda \frac{\partial^{2} T(t, x)}{\partial x^{2}}-\frac{\partial F(t, x)}{\partial x}
$$

where $F(x, t)$ is the total radiative flux. By considering $\lambda=\alpha \rho c_{\mathrm{p}}$, Eq. (4) is described as follows:

$$
\frac{\partial T}{\partial t}=\alpha \frac{\partial^{2} T}{\partial x^{2}}-\frac{\alpha}{\lambda} \frac{\partial F}{\partial x}
$$

It is obvious that $T$ and $F$ are intensive properties that depend on the time and location. Hereafter we abbreviate the notations, for example, $T(x, t)$ is written as $T$ or $T(x)$. The total radiative flux $F$ is derived from the generation and absorption of radiation. The radiative energy emitted from a semi-transparent volume $d V$ is given by

$$
n^{2} \kappa I_{\mathrm{b}}(T) d V
$$

where $I_{\mathrm{b}}(T)$ represents the radiation intensity of a black body; $n$, the refractive index; and $\kappa$, the absorption coefficient of the slag.

According to Lambert's law, the change of radiation intensity, $d I$, is proportional to the path length in the radiation of the semi-transparent material, $d l$, and radiation intensity, I.

$$
d I=-\kappa I d l
$$

The radiation and the absorption of an infinitesimal volume $d V$ are isotropic. The radiation intensity $I$ in the direction making an angle $\psi$ with a normal to the layer is given by 


$$
\mu \frac{d I}{d x}=\kappa\left\{n^{2} I_{\mathrm{b}}(T)-I\right\}
$$

where $\mu=\cos \psi$. Integration of Eq. (8) for $x$ gives

$$
\begin{aligned}
I(x)^{+}= & I(0)^{+} \exp \left(-\frac{\kappa x}{\mu}\right) \\
& +\exp \left(-\frac{\kappa x}{\mu}\right) \int_{0}^{x} \kappa I_{\mathrm{b}}\left(x^{\prime}\right) \exp \left(-\frac{\kappa x^{\prime}}{\mu}\right) \frac{d x^{\prime}}{\mu}
\end{aligned}
$$

and

$$
I(x)^{-}=\exp \left(-\frac{\kappa x}{\mu}\right) \int_{x}^{\infty} \kappa I_{\mathrm{b}}\left(x^{\prime}\right) \exp \left(-\frac{\kappa x^{\prime}}{\mu}\right) \frac{d x^{\prime}}{\mu} .
$$

where $I^{+}$represents the radiation traveling in the positive $x$ direction; $I^{-}$, the radiation traveling in the negative $x$ direction; and $I(0)^{+}$, the radiation in the positive direction at the boundary between the platinum crucible and slag layer. The radiation intensity at the surface of the platinum crucible attached to the slag layer is given by $\varepsilon n^{2} I_{b}(T(0))$. $\varepsilon$ represents the emissivity of the surface of the platinum crucible. $I_{\mathrm{b}}(T(0))$ represents the intensity of black body radiation determined by the temperature at $x=0$.

The platinum surface reflects a portion of the incoming radiation from the slag layer and absorbs the remaining portion. The reflection ratio for the incident radiation $I(0)^{-}$ traveling in the negative $x$ direction at $x=0$ is $1-\varepsilon$.

The required boundary conditions $I(0)^{+}$in Eq. (9) on the radiation is given as the radiation traveling in the positive $x$ direction at $x=0$ as follows:

$$
I(0)^{+}=\varepsilon n^{2} I_{\mathrm{b}}(T(0))+(1-\varepsilon) I(0)^{-}
$$

Since the radiation intensity is a function of the direction as shown in Eq. (8), heat flux is derived by hemispherical integration of the radiation intensity with the $x$ axis as the polar axis. In the case of the radiative heat flux crossing the area parallel to the slag layer, $F(x)$ is given by

$$
F(x)=\int_{0}^{2 \pi} d \phi \int_{0}^{\pi / 2} I(x, \mu) \sin \psi d \psi
$$

In order to simplify the equations, we employed the following approximations: all physical properties of the slag layer and platinum crucible are assumed to be constant over the optical wavelength, i.e., gray approximation, and over angle $\psi$ Since the hemispherical total emissive power of a blackbody radiating into vacuum, $E(x)$, is defined by

$$
E(x)=\int_{0}^{2 \pi} d \phi \int_{0}^{\pi / 2} I_{\mathrm{b}} d \psi
$$

and $\sin \psi d \psi=-d \mu, F(x)$ is given by

$$
\begin{aligned}
F(x) & =2 n^{2} E(0) R(x)+2 n^{2} \kappa(1-\varepsilon) \int_{0}^{\infty} E\left(x^{\prime}\right) R\left(x+x^{\prime}\right) d x^{\prime} \\
& +2 n^{2} \kappa\left\{\int_{0}^{x} E\left(x^{\prime}\right) R\left(x-x^{\prime}\right) d x^{\prime}-\int_{x}^{\infty} E\left(x^{\prime}\right) R\left(x^{\prime}-x\right) d x^{\prime}\right\}
\end{aligned}
$$

where

$$
R(x)=\int_{0}^{1} \exp (-\kappa x / \mu) d \mu
$$

\subsection{At Boundary between the Slag Layer and Cru- cible}

The slag layer is assumed to be in good contact with the platinum crucible. Any thermal resistance at the interfaces is neglected because of good wettability between the platinum crucible and slag. Then, the following two conditions should be satisfied at the boundary: (1) continuity of temperature; (2) continuity of heat flux. These conditions are described as follows:

$$
\begin{aligned}
& \left.T\right|_{0-}=\left.T\right|_{0+} \\
& -\left.\lambda_{\mathrm{d}} \frac{d T}{d x}\right|_{0^{-}}=-\left.\lambda_{\mathrm{s}} \frac{d T}{d x}\right|_{0^{+}}+F(0)
\end{aligned}
$$

where the subscripts, $0^{-}$and $0^{+}$, refer to a negative direction at $x=0$, i.e., the region in the platinum crucible, and a positive direction at $x=0$, i.e., the region in the slag layer, respectively.

\subsection{In the Crucible}

In the platinum crucible, $F$ is zero. Equation (5) is reduced to

$$
\frac{\partial T}{\partial t}=\alpha \frac{\partial^{2} T}{\partial x^{2}}
$$

\subsection{At the External Surface of the Crucible}

The heat was lost from the external bottom surface, at $x=-l_{\mathrm{d}}$, to the surroundings according to the radiative law

$$
\left.\lambda \frac{d T}{d x}\right|_{-l_{\mathrm{d}}}=4 \varepsilon \sigma T_{0}^{3}\left(T-T_{0}\right)
$$

where $T_{0}$ represents the ambient temperature. $T_{0}$ is equal to the initial sample temperature before laser irradiation. $\sigma$ represents the Stefan-Boltzmann constant.

\section{Numerical Solution}

Before laser pulse irradiation, the net heat flow in the cell is zero. Since the temperature increase $\theta(x, t)$ caused by the laser pulse is small compared to $T_{0}$, the net radiation intensity at any point is linearized with respect to $\theta$. For net radiative heat flow in Eq. (14), the total emissive power of a blackbody is reduced to

$$
E=4 \sigma T_{0}^{3} \theta
$$

\subsection{Discretization}

Appropriate thickness of slag, $l_{\mathrm{s}}$, is assumed for the slag layer to satisfy the semi-infinite boundary condition. The platinum crucible layer is divided into $n_{\mathrm{d}}$ intervals, and the slag layer is devided into $n_{\mathrm{s}}$. The representative points are placed on each boundary of the layer. The corresponding number of representative points $i$ and locations is as fol- 
lows:

$$
\begin{array}{lll}
\text { external surface } & x=-l_{d} & i=0 \\
\text { in the platinum crucible } & x<-l_{d}<0 & 1 \leq i \leq n_{\mathrm{d}}-1 \\
\text { crucible/slag boundary } & x=0 & i=n_{\mathrm{d}} \\
\text { in slag layer } & 0<x & n_{\mathrm{d}}+1 \leq i \leq n_{\mathrm{d}}+n_{\mathrm{s}}
\end{array}
$$

The temperature in the platinum crucible and slag layer are presented by a set of finite difference equations. Eqs. (5) and (18) are iteratively solved by successive over-relaxation (SOR). ${ }^{22)}$ For a position $i$ at time $t=j \Delta t$ temperature $\theta_{i, j}$ for the $(m+1)$ th iterate is

$$
\begin{aligned}
\theta_{i, j}^{(m+1)}= & \theta_{i, j}^{(m)}+\omega\left[\frac{r}{2(1+r)}\left\{\theta_{i-1, j}^{(m+1)}+\theta_{i+1, j}^{(m+1)}\right\}\right. \\
& \left.-e_{\mathrm{s}} \frac{r}{2(1+r) \lambda}\left(\frac{\partial F}{\partial x}\right)_{i, j}+\frac{B_{i, j-1}}{(1+r)}-\theta_{i, j}^{(m)}\right] \ldots \ldots
\end{aligned}
$$

where

$$
\begin{aligned}
& e_{\mathrm{s}}= \begin{cases}1 & \text { in slag } \\
0 & \text { in crucible }\end{cases} \\
& B_{i, j-1}=\theta_{i, j-1}+\frac{r}{2}\left(\theta_{i-1, j-1}-2 \theta_{i, j-1}+\theta_{i+1, j-1}\right) \\
& -e_{\mathrm{s}} \frac{\alpha \Delta t}{\lambda}\left(\frac{\partial F}{\partial x}\right)_{i, j} \\
& r=\frac{\alpha \Delta t}{(\Delta x)^{2}}
\end{aligned}
$$

$\Delta x$ is spatial interval and assumed to be uniform for each layer and $\Delta t$ is the time mesh size. The relaxation factor $\omega$ is determined to achieve fast convergence. At the boundary between the platinum crucible and slag layer, differentiating Eq. (17) with respect to $x$ gives

$$
\theta_{n_{\mathrm{d}}, j}=\left(\lambda_{\mathrm{d}} \frac{\theta_{n_{\mathrm{d}}-1}}{\Delta x_{\mathrm{d}}}+\lambda_{\mathrm{s}} \frac{\theta_{n_{\mathrm{d}}+1}}{\Delta x_{\mathrm{s}}}+F(0)\right) /\left(\frac{\lambda_{\mathrm{d}}}{\Delta x_{\mathrm{d}}}+\frac{\lambda_{\mathrm{s}}}{\Delta x_{\mathrm{s}}}\right)
$$

The temperature at the boundary $\theta_{n_{\mathrm{d}} j}$ is represented by a single point corresponding to Eq. (16). By differentiating Eq. (19) at the external surface, we have

$$
\theta_{0, j}=\frac{\theta_{1, j}}{1+\left(\lambda_{\mathrm{d}} / 4 \varepsilon \sigma T_{0}^{3} \Delta x_{\mathrm{d}}\right)}
$$

where $\Delta x_{\mathrm{d}}=l_{\mathrm{d}} / n_{\mathrm{d}}$ and $\Delta x_{\mathrm{s}}=l_{\mathrm{s}} / n_{\mathrm{s}}$.

It is difficult to determine the thickness of the slag layer that is considered to be infinity for calculation for combined heat transfer. The condition is numerically evaluated as follows. Two temperatures are calculated at the end point of the slag layer, $n_{\mathrm{d}}+n_{\mathrm{s}}$, by two equations for comparison. The one is

$$
\theta_{n_{\mathrm{d}}+n_{\mathrm{s}}}=\theta_{n_{\mathrm{d}}+n_{\mathrm{s}}-1}
$$

for adiabatic; the other is
Table 1. The physical properties of slag for calculation.

\begin{tabular}{ccccc}
\hline Condition & $T_{0}(\mathrm{~K})$ & $\lambda_{s}\left(\mathrm{~W} \mathrm{~s}^{-1} \mathrm{~K}^{-1}\right)$ & $\alpha_{s}\left(\times 10^{-7} \mathrm{~m}^{2} / \mathrm{s}\right)$ & $n$ \\
\hline 1 & 1743 & 1.53 & 4.67 & 1.5 \\
2 & 1583 & 1.56 & 4.66 & 1.5 \\
3 & 1743 & 2.36 & 7.62 & 1.5 \\
4 & 1583 & 2.80 & 8.94 & 1.5 \\
\hline
\end{tabular}

$$
\theta_{n_{\mathrm{d}}+n_{\mathrm{s}}}=0
$$

for isothermal. The resultant differences of the temperature rise at the surface of the platinum layer, $\theta_{0}$, for any time and any condition in this study are less than $10^{-4} \%$ of the values. The results confirm that the thickness of slag layer is appropriate.

The field is presumed to be swept with $i$ increasing from zero to $n_{\mathrm{d}}+n_{\mathrm{s}}$, so that the $\theta$ value at iterative level $(m+1)$ is available at $i-1 .(d F=d x)_{i, j}$ is numerically calculated by Eqs. (20), (14), and (15) from calculated temperature distribution $\theta$ for each step.

\subsection{Physical Properties and Calculation Conditions}

The physical parameters of platinum ${ }^{23)}$ are used for the platinum crucible employed as follows: $l_{\mathrm{d}}=2.0 \times 10^{-4} \mathrm{~m}$, $\alpha_{\mathrm{d}}=2.4 \times 10^{-5} \mathrm{~m}^{2} \mathrm{~s}^{-1}, \lambda_{\mathrm{d}}=70.56 \mathrm{~W} \mathrm{~m}^{-1} \mathrm{~K}^{-1}$, and $\varepsilon=0.2$. The parameters of the slags are chosen for four cases. The physical properties are given in Table 1, where $n$ is the refractive index of the slag and $T_{0}$ is the measured temperature. The physical properties and the temperature range are determined to cover those of the slag samples measured by this method. ${ }^{14,15,19,20)}$ The maximum temperature was limited by the heater of the equipment. The minimum is determined by the melting point of the samples.

The conditions for the numerical calculation are set as follows. The time mesh size, $\Delta t$, is $10^{-5}$. For fast convergence, the relaxation factor, $\omega$, is set to 1.6. Error tolerance for each point is set to $10^{-6}$. If the iteration-to-iteration absolute difference $\left|\theta_{i, j}^{(m)}-\theta_{i, j}^{(m-1)}\right|$ is less than the tolerance, it is considered to be convergent. The thickness of the slag layer, $l_{\mathrm{s}}$, is $2 \times 10^{-3} \mathrm{~m}$. The number of meshes for the slag layer, $n_{\mathrm{s}}$, is 400 , and that for the platinum crucible, $n_{\mathrm{d}}$, is 16 .

\subsection{Initial Condition}

This technique assumes instantaneous heating and uniform temperature in a platinum layer. The laser pulse is given as a simple initial temperature distribution: the temperature of the platinum layer is 1 and that of the slag layer is zero. The initial temperature distribution is given as

$$
\theta_{i, 0}= \begin{cases}1 & 0 \leq i \leq n_{\mathrm{d}} \\ 0 & n_{\mathrm{d}}+1 \leq i \leq n_{\mathrm{s}}+n_{\mathrm{d}}\end{cases}
$$

\section{Result and Discussion}

The cases without any radiative effect were calculated by setting all radiation terms in the equations to zero to evaluate the effect of radiation. Figure 4 shows the calculated temperature responses for the case with and without radiative effect and those determined by using a fitting proce- 


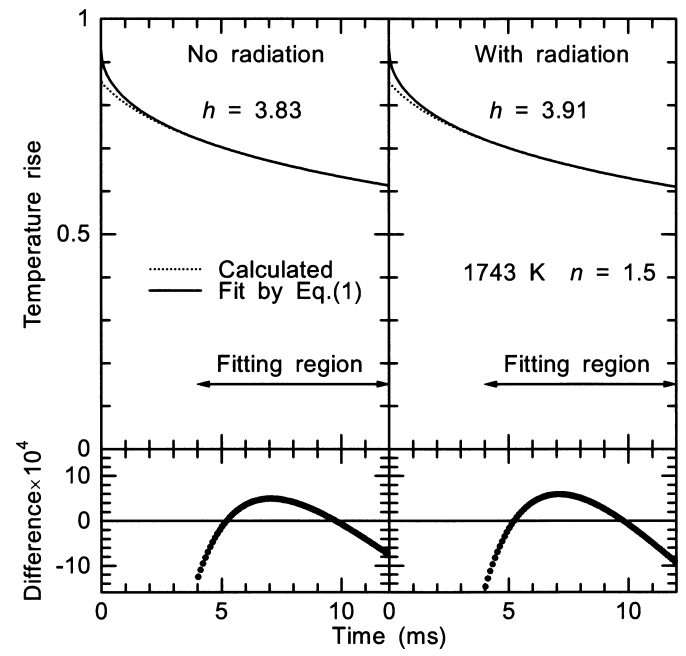

Fig. 4. Numerically calculated temperature response with and without radiative heat flux. The response determined by fitting procedure to Eq. (1) and difference of two curves is also plotted. The thermal conductivity of slag, $\lambda_{\mathrm{s}}$, and The thermal diffusivity, $\alpha_{\mathrm{s}}$ are $1.53 \mathrm{~W} /(\mathrm{s} \cdot \mathrm{K})$ and $4.67 \times$ $10^{-7} \mathrm{~m}^{2} / \mathrm{s}$, respectively. $h$ show the values derived by fitting to Eq. (1).

Table 2. The values derived from physical properties by Eq. (1) and those obtained by curve fitting to Eq. (2) on numerically calculated temperature responses without radiative effect.

\begin{tabular}{ccc}
\hline $\begin{array}{c}\text { Calculation } \\
\text { condition }\end{array}$ & $\begin{array}{c}\text { From physical } \\
\text { properties }\end{array}$ & $\begin{array}{c}\text { By curve } \\
\text { fitting }\end{array}$ \\
\hline 1 & 3.81 & 3.83 \\
2 & 3.89 & 3.91 \\
3 & 4.60 & 4.61 \\
4 & 5.04 & 5.04 \\
\hline
\end{tabular}

dure to Eq. (1). The fitting regions is set as same as the experimental procedure to avoid the effects of the laser duration and the temperature distribution in the platinum layer. ${ }^{15)}$ Each calculated response agrees with Eq. (1).

Table 2 shows the $h$ values derived by the fitting procedure on calculated temperature response without considering radiation. The $h$ values derived from the physical properties of the slag and the crucible by Eq. (2) are also shown in the table. The good agreement for each condition shows the validity of the present calculation. We can then evaluate the magnitude of the effect of radiation on measured values by comparison of the calculated responses with or without radiative heat transfer.

Figure 5 shows the differences of responses in the fitting time region, 4 to $12 \mathrm{~ms}$. The effect of radiation increases with time and initial temperature and decreases with the thermal conductivity of slag and absorption coefficient. Four absorption coefficients of slag, $\kappa$ of 10, 100, 1000, and $5000 \mathrm{~m}^{-1}$ are employed. The mode of radiative heat transfer depends on $\kappa$ and the characteristic length of the sample, $L$. The characteristic length corresponds to the thickness of the region of the temperature gradient. It is about $10^{-3} \mathrm{~m}$ for this case. The mode of heat transfer depends on the value of the optical thickness $\kappa L$. If $\kappa L \ll 1$,

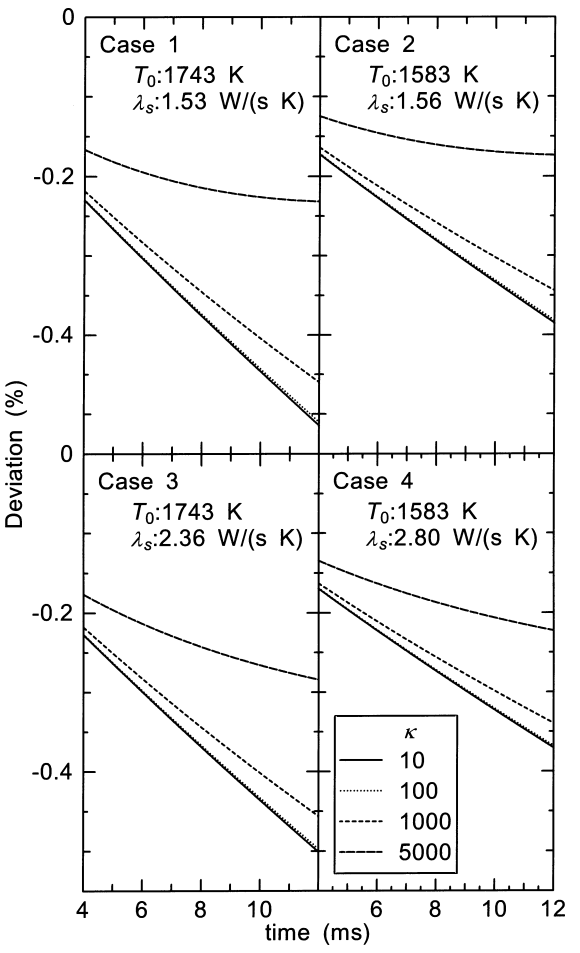

Fig. 5. The deviations of numerically calculated temperature responses from those without radiative heat flow.

Table 3. Ratios of $h$ values including radiative heat transfer to ones omitting radiative effect derived from calculated temperature response curves.

\begin{tabular}{ccccc}
\hline \multirow{2}{*}{ Calculation } & \multicolumn{4}{c}{$\kappa\left(\mathrm{m}^{-1}\right)$} \\
\cline { 2 - 5 } condition & 10 & 100 & 1000 & 5000 \\
\hline 1 & 1.0210 & 1.0207 & 1.0181 & 1.0051 \\
2 & 1.0155 & 1.0153 & 1.0133 & 1.0038 \\
3 & 1.0184 & 1.0182 & 1.0162 & 1.0076 \\
4 & 1.0129 & 1.0128 & 1.0115 & 1.0059 \\
\hline
\end{tabular}

the radiative heat flux does not depend on $\kappa$ because the slag is supposed to be transparent. Since the difference of the temperature responses for the cases of $\kappa=10$ and 100 is small, it could be concluded that the case of $\kappa=10$ is as transparent. When $\kappa L$ is close to unity, we can then derive the temperature responses only numerically. When $\kappa L \gg 1$, optically thick approximation or diffusion approximation, is valid. The radiative heat transfer reduced to become the same mode as conductive heat transfer, which is proportional to the gradient of temperature distribution. The proportional coefficient for radiative heat transfer is proportional to the inverse of $\kappa$. Then, above $5000 \mathrm{~m}^{-1}$ of $\kappa$, we could assume that the effect of radiation monotonously decreased.

The effect of radiation on $h$ values is evaluated as follows: $h$ values are derived by a fitting procedure for all temperature responses in Fig. 5. For each case with radiation, the ratio of $h$ value with radiation and those without radiation are shown in Table 3. Similar tendencies as those in Fig. 5 are also noted for the values in Table 3. The effect of $\kappa$ is monotonous. From the above mentioned discussion on absorption coefficient, we conclude that the maximum dif- 
ference on $h$ caused by radiative heat transfer is $2.2 \%$ for any value of $\kappa$.

From Eq. (2) and Table 3, we conclude that the magnitude of the propagated error on thermal diffusivity or conductivity by radiation is therefore less than $4.4 \%$.

Here, we summarize the effect of radiative heat transfer on the measured value by conventional front-heating reardetection laser flash method for comparison. Ohta et al. developed a differential three-layered laser flash method for melts at high temperatures using a cell with a mechanism to precisely change the thickness of liquid and employed it for molten materials. ${ }^{5-9)}$ This system was recently also employed by other researchers. ${ }^{10-12)}$ In this method, the effect of radiation is a complicated function of the thickness of the sample, the change of the thickness for differential measurement, refractive index and absorption coefficient of slag, thermal conductivity, and temperature of sample. The function can only be solved numerically. The effect is rather large even at relatively low temperature. The effect is also known to be non-monotonous and sensitive for absorption coefficient. In the case of a transparent slag at $1500 \mathrm{~K}$, the value derived by this technique is $20 \%$ larger than that with a correction for radiation. Near the transparent region of the absorption coefficient, the values increase with increasing absorption coefficient. Then, for a semi-transparent slag such as that containing Fe ions, the effect of radiation is approximately twice as large as that for the transparent case. If the absorption coefficient is sufficiently large as discussed previously, the effect of radiation decreases with increase in absorption coefficient, according to optically thick approximation, or diffusion approximation,

Ohta et al. were successful in correcting the effect of radiation $^{6-8)}$ with the measurement of the optical properties of the slag for the calculation of the radiative heat transfer. ${ }^{13)}$ In their study, a strong positive dependence on temperature of the slag containing Fe ions was observed for the thermal conductivity without the correction of radiative heat. This tendency differs from that shown by the other slag having similar composition. However, the corrected data shows no obvious temperature dependence as similar to that for the other slag with similar composition without including $\mathrm{Fe}$ ions. This result clearly shows that the correction is indispensable for measurement by a three-layered method. However, the calculation is not easy and the optical properties essential for correction are usually not available.

\section{Conclusion}

We have developed front-heating front-detection laser flash method using a short initial time period for the measurement of molten slag at high temperatures. In this paper, we revealed that the effect of radiation on this new technique is very small compared with that on the three-layered technique. At $1500 \mathrm{~K}$, the effect of radiation on the measured value is 20 to $40 \%$ for the three-layered technique. The effect is sensitive to experimental conditions and physical properties of slag such as absorption coefficient. The complicated procedure of correction is indispensable. Meanwhile, the effect of radiation on measured value for the newly developed technique is less than $3.1 \%$ at $1583 \mathrm{~K}$ and less than $4.4 \%$ even at $1743 \mathrm{~K}$. The effect monoto- nously decreases with increasing absorption coefficient of slag. With the easy operation and data processing, this technique is considered to be versatile for the measurement of silicate melts at high temperature.

$\begin{array}{rlr}\text { Nomenclature } & \\ b: & \text { Thermal effusivity } & \left(\mathrm{J} \mathrm{s}^{-1 / 2} / \mathrm{m}^{2} \cdot \mathrm{K}\right) \\ c_{\mathrm{p}}: & \text { Specific heat } & (\mathrm{J} / \mathrm{kg} \cdot \mathrm{K}) \\ F: & \text { Total radiative flux } & \left(\mathrm{W} / \mathrm{m}^{2}\right) \\ h: & b_{\mathrm{s}} /\left(\rho_{\mathrm{d}} c_{\mathrm{pd}} l_{\mathrm{d}}\right) & \left(\mathrm{s}^{-1 / 2}\right) \\ I: & \text { Radiation Intensity } & \left(\mathrm{W} / \mathrm{m}^{2} \cdot \mathrm{sr}\right) \\ I_{\mathrm{b}}: & \text { Radiation intensity of a black body } & \left(\mathrm{W} / \mathrm{m}^{2} \cdot \mathrm{sr}\right) \\ l: & \text { Thickness of layer } & (\mathrm{m}) \\ L: & \text { Characteristic length for radiation } & (\mathrm{m}) \\ n: & \text { Refractive index } & (-) \\ n_{\mathrm{s}}: & \text { Number of meshes for slag layer } & (-) \\ n_{\mathrm{d}}: & \text { Number of meshes for platinum layer } & (-) \\ t: & \text { time } & (\mathrm{s}) \\ T: & \text { Temperature } & (\mathrm{K}) \\ T_{0}: & \text { Ambient temperature } & (\mathrm{K}) \\ x: & \text { Position } & (\mathrm{m})\end{array}$

Greek letters

$\alpha$ : Thermal diffusivity $\quad\left(\mathrm{m}^{2} / \mathrm{s}\right)$

$\varepsilon$ : Emissivity of the surface of the platinum crucible

$\kappa:$ Absorption coefficient of slag $\left(\mathrm{m}^{-1}\right)$

$\lambda$ : Thermal conductivity

$\theta$ : Temperature rise

$\theta_{\mathrm{M}}$ : Maximum temperature rise of the irradiated surface

$\rho:$ Density

$\sigma:$ Stefan-Boltzmann constant

$\left(\mathrm{kg} / \mathrm{m}^{3}\right)$

$\omega$ : Relaxation factor for the SOR

$\left(\mathrm{W} / \mathrm{m}^{2} \cdot \mathrm{K}^{4}\right)$

Subscript

$d$ : Platinum layer

$s$ : Slag layer

Superscript

+ : Traveling in the positive $x$ direction

- : Traveling in the negative $x$ direction

\section{REFERENCES}

1) W. J. Parker, R. J. Jenkins, C. P. Butler and G. L. Abbott: J. Appl. Phys., 32 (1961), 1679.

2) J. L. Bates: Thermal Diffusion and Electrical Conductivity of Molten and Solid Slags, Battelle Pacific Northwest Lab. Rep., (1976).

3) M. M. Farooq, W. H. Giedt and N. Araki: Int. J. Thermophys., 2 (1981), 39

4) M. I. Darby: High Temp. High Press., 15 (1983), 629.

5) H. Ohta, G. Ogura, Y. Waseda and M. Suzuki: Rev. Sci. Instrum., 61 (1990), 2645

6) Y. Waseda, M. Masuda and H. Ohta: Proc. of the 4th Int. Symp. on Advanced Nuclear Energy Research, Japan Atomic Energy Research Institute, Kashiwa, Chiba, (1992), 298.

7) H. Ohta, M. Masuda, K. Watanabe, K. Nakajima, H. Shibata and Y. Waseda: Tetsu-to-Hagané, 80 (1994), 463.

8) Y. Waseda, M. Masuda, K. Watanabe, H. Shibata, K. Nakajima and H.Ohta: High Temp. Mater. Proc., 13 (1994), 267.

9) Y. Maeda, H. Sagara, R. P. Tye, M. Masuda, H. Ohta and Y. Waseda: Int. J. Thermophys., 17 (1996), 253.

10) W-Q. Jin, T. Nagashima, S. Yoda, X-A. Liang, Z-L. Pang: Chin. Phys. Lett., 19 (2002), 569.

11) R. Eriksson, M. Hayashi and S. Seetharaman: Int. J. Thermophys., 24 (2003), 785.

12) R. Eriksson and S. Seetharaman: Metall. Mater. Trans. B, 35B 
ISIJ International, Vol. 46 (2006), No. 3

(2004), 461.

13) H. Ohta, K. Watanabe, K. Nakajima and Y. Waseda: High Temp. Mater. Proc., 12 (1993), 139.

14) H. Ohta, H. Shibata, A. Suzuki and Y. Waseda: Symposium Fundamentals of Metallurgical Processing, ed. by C. Kaiura, C. Pickels, T. Utigard and A. Vahed, METSOC, Canada, (2000), 19.

15) H. Ohta, H. Shibata, A. Suzuki and Y. Waseda: Rev. Sci. Instrum., 72 (2001), 1899.

16) Y. Tada, M. Harada, M. Tanigaki and W. Eguchi: Rev. Sci. Instrum., 49 (1978), 1305.

17) Y. Tada, M. Harada, M. Tanigaki and W. Eguchi: Ind. Eng. Chem. Fundam., 20 (1981), 333.
18) T. Azumi and Y. Takahashi: Rev. Sci. Instrum., 52 (1981), 1411.

19) S. Inaba, Y. Kimura, H. Shibata and H. Ohta: ISIJ Int., 44 (2004), 2120.

20) H. Shibata, A. Suzuki and H. Ohta: Mater. Trans., 46 (2005), 1877.

21) R. Siegel and J. R. Howell: Thermal Radiation Heat Transfer, Hemisphere, Washington, (2002), 419.

22) T. Saitoh: Computer-Aided Heat Transfer, Youkendo, Tokyo, (1986), 81 .

23) Y. S. Touloukian, R. W. Powell, C. Y. Ho and M. C. Nicolaou: Thermophysical Properties of Matter., Thermal Diffusivity, TPRC Data Services 19, Plenum Press, New York, (1973), 135. 